\title{
The new EN ISO 52000 family of standards to assess the energy performance of buildings put in practice
}

\author{
Dick van Dijk ${ }^{1, *}$, and Jaap Hogeling ${ }^{1}$ \\ ${ }^{1}$ EPB Center, Weena 505, 3013 AL Rotterdam, The Netherlands
}

\begin{abstract}
The set of Energy Performance of Buildings (EPB) standards, developed under a mandate from the European Commission to support the EPBD, has been published in summer 2017. This set of standards enables to assess the overall energy performance of a building. A number of key EPB standards are available at global level (the EN ISO 52000 family of standards), while others are for the moment only available at European level (CEN standards). The revised European Directive on the Energy Performance of Buildings (EPBD), published in June 2018, obliges the EU Member States to describe their national calculation methodology following the 'national annexes' of the so called 'overarching' EPB standards. This will force the Member States to explain where and why they deviate from these standards and will lead to an increased recognition and promotion of the set of EPB standards across Europe and beyond. This paper provides background information and tips on how to put the EN ISO 52000 family of standards into practice.
\end{abstract}

\section{Introduction}

The set of Energy Performance of Buildings (EPB) standards has been published in summer 2017. This set of standards enables to assess the overall energy performance of a building; in most cases, but not exclusively, by calculation. A number of key EPB standards are available at global level (the EN ISO 52000 family of standards), while others are for the moment only available at European level (CEN standards).

The revised European Energy Performance of Buildings Directive (EPBD:2018 [1]) requires (in Annex I):

"Member States shall describe their national calculation methodology following the national annexes of the overarching standards, namely ISO 52000-1, 52003-1, 52010-1, 52016-1, and 52018-1, developed under mandate M/480 given to the European Committee for Standardisation (CEN). This provision shall not constitute a legal codification of those standards."

So, although the new EPBD does not force the Member States to apply the set of EPB standards, the obligation to describe the national calculation methodology following the national annexes of the overarching standards will push the Member States to explain where and why they deviate from these standards. This will lead to an increased recognition and promotion of the set of EPB standards across the Member States and beyond.
In the following, background information and tips are given on how to put the EN ISO 52000 family of standards into practice.

\section{The set of EPB standards}

\subsection{Mandate from European Commission to support EPBD}

The set of standards and accompanying technical reports on the energy performance of buildings (set of EPB standards) have been prepared under a mandate, given to CEN by the European Commission and the European Free Trade Association, to support the EPBD (Mandate M/480, 2011 - 2016) [2].

Figure 1 shows a simplified flow chart of the main modules or elements in the assessment of the energy performance of buildings. Each of these modules is covered by one or more EPB standards.

\subsection{Overall consistency}

As figure 1 illustrates, the input needed in one EPB standard is provided as output by another EPB standard and, eventually, by product data (e.g. component properties), project data (e.g. geometry) and boundary conditions (e.g. climatic data, conditions of use). Evidently, it is extremely important that overall consistency is secured and maintained in terms, definitions and symbols and in the overall approach.

The teams who were responsible for the development of the set of EPB standards collectively worked out a set of quality documents, rules and templates (with the basis

Corresponding author: dick.vandijk@epb.center 
formed by [3], [4]). These quality documents were immediately put into practice during the development of the standards and are, based on the experience, gradually (being) updated.

A complete overview of all EPB standards is given at the EPB Center website (www.epb.center, [5]). More background information can also be found in recent articles, e.g. in the REHVA Journal ([6], [7]).

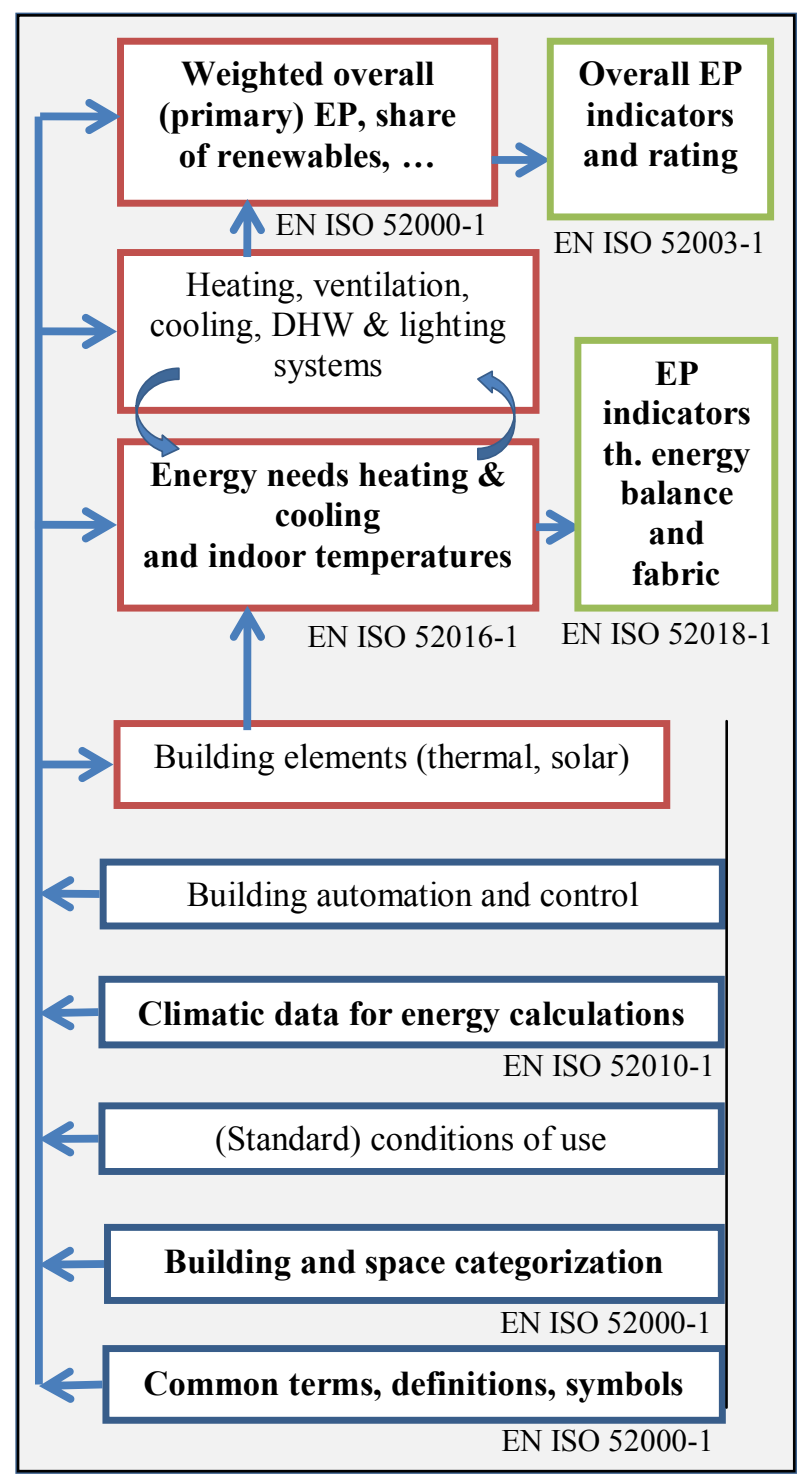

Fig. 1. Flow chart of the set of EPB standards with position of the five 'overarching' EPB standards (in bold).

\subsection{Holistic approach: instrument for policy targets and driver for innovation}

In the past, energy performance requirements were set at component level - minimum thermal insulation levels and minimum efficiencies of products. This, however, leads to sub-optimal solutions and creates a barrier to the necessary technology transitions.

The set of EPB standards is based on the holistic or systemic approach: the assessment of the overall energy performance of a building. This implies that all types of building related energy uses (heating, lighting, cooling, air conditioning, ventilation) and outdoor climatic and local conditions, as well as indoor climate requirements are considered, including the sometimes complex and dynamic interactions between these various aspects.

This also implies that any combination of technologies can be used to reach the intended overall energy performance level, at the lowest cost. Due to this 'competition' between different technologies, the holistic approach is a key driver for technological innovation and change. Countries using the approach for several years take, for instance, The Netherlands - have experienced large scale implementation and cost savings on a variety of new technologies.

The holistic approach is a key instrument to set and evaluate policy targets. Clear and consistent policy targets play an important role in driving innovation in the building sector ([8]).

\subsection{The five 'Overarching standards mentioned in EPBD}

The revised EPBD, quoted in the Introduction above, mentions explicitly five so called 'overarching' standards:
EN ISO 52000-1 [9],
EN ISO 52003-1 [10],
EN ISO 52010-1 [11],
EN ISO 52016-1 [12], and
EN ISO 52018-1 [13].

Figure 1 (roughly) shows which elements are covered by these standards (bold and numbered). These five 'overarching' EPB standards have in common that each of them describes an important step in the assessment of the energy performance of building.

It is very fortunate that these five EPB standards are also available as ISO standards, which creates a strong basis for the roll-out of the other (CEN) EPB standards at worldwide level as well [14]. Harmonization of EPB assessment methodologies at global level will strengthen innovation on EPB solutions and products.

\section{Supporting documents and tools}

\subsection{Accompanying Technical Reports}

Each EPB standard needs to be as concise and unambiguous as possible: fit to be implemented or referenced in national or regional building codes. For that reason each EPB standard contains purely normative procedures, with only brief notes (not part of the normative text) where unavoidable.

However, there would be a risk that the purpose and limitations of the EPB standards would be misunderstood, if the background and context to their contents - and the thinking behind them - would not be explained in some detail to readers of the standards. Therefore, each EPB standard (or sometimes a cluster of EPB standards) is accompanied by an (informative) 
technical report in which such kind of information is made available (including calculation examples), to properly understand, apply and nationally or regionally implement the EPB standards.

\subsection{Accompanying spreadsheets}

One of the instruments to check the necessary overall consistency and coherence, in terminology, approach, input/output relations and formats, was the preparation of a spreadsheet for each EPB calculation standard. These spreadsheets, prepared on the basis of a common template, intend to demonstrate the correctness of the EPB calculation procedures and to enable a check of the list of input and output variables; in particular with respect to the interconnections between the standards, as mentioned above.

Because each spreadsheet was developed in parallel with the corresponding EPB standard, and was used to detect omissions in the standard and mismatches in input-output relations between the EPB standards, the most recently available version of the spreadsheet in many cases reflects a draft version of the standard (from 2014 or 2015) and has not or not yet been updated to the level of the published version (summer 2017) of the standard. These spreadsheets can be downloaded from the EPB Center website.

\subsection{More to come...}

In the context of a Service Contract [17] with the European Commission, the EPB Center intends to upgrade the spreadsheets of the most important standards during 2019 and 2020 and prepare case studies. See more under section 6 of this paper.

\section{National Annexes}

\subsection{National choices to tailor the EPB standards to the national situation}

The set of EPB standards offers an internationally agreed set of methods to assess the energy performance of buildings in a harmonized, modular and transparent way. At the same time, the individual countries can tailor the standards to their national building regulations, building tradition, technology infrastructure and climate. They thus combine the benefits of an internationally harmonized approach with specific national or regional features.

For this purpose, each EPB standard has an "Annex A": a template providing choices:

- choices between specific methods (e.g. simple or more detailed),

- including choices of references to other EPB or national standards for specific elements of the calculation, and

- choices of input data; these cover technical, policy related or climate related data.
The choices according to the Annex A template, made by the Member States and/or National Standards Bodies, are to be laid down in National Datasheets (e.g. annexed to the building code) or in National Annexes to these standards (referenced by the building code). In any case, each of these National Datasheets or National Annexes shall comply with the template of Annex A of the corresponding EPB standard.

In the context of the afore mentioned Service Contract with the European Commission [17], the EPB Center will collect and distribute examples and tips on the preparation of National Annexes. And also provide support on how to use the template of Annex A to report a (deviating) national methodology, as requested in Annex I of the revised EPBD [1], as quoted in the Introduction above.

\subsection{Examples of the types of choices}

A rough impression of the types of choices provided in the EPB standards can be obtained by looking at the main types of choices provided in the five 'overarching' EPB standards:

EN ISO 52000-1 (On the general EPB framework, [9]): About 30 tables with choices. For instance on:

- Physical parameters (e.g. gross calorific values).

- Differentiation into different building and space categories (distinction between -for example- single family house, apartment building, office, hospital, education, assembly, sport, restaurant, hotel, holiday home, etc.; or e.g. a less refined differentiation).

Plus related issues such as: which categories are kept outside the boundaries of the EPB-assessment (for instance industrial sites, workshops, indoor parking; or any other choice of building or space categories).

This categorization has a strong influence on the EP assessment: each category is linked to an assumed set of conditions of use (temperature, IAQ, DHW, lighting, ...). By the way, the (assumed, standard) settings for these conditions of use are also determined nationally, in the relevant other EPB standards; see figure 1.

$\mathrm{V}$ ia these conditions of use, the categorization has also a strong effect on the need to partition the building into different zones or sections for the calculation: e.g. spaces with different temperature settings may require separate calculation (separate thermal zones). This, in turn, leads to more input data (e.g. the floor, façade and window area per zone).

Moreover, the more refined the categorization and the distinction in conditions of use, the more likely it is that the minimum EP requirements need to be refined (see also EN ISO 52003-1 below).

But on the other hand, if the categorization is less refined, the predicted energy performance may be less close to the energy performance in practice.

- Energy performance boundaries (e.g. whether $P V$ surplus to the grid is rewarded in the energy performance or not. Or whether "distant" and/or 
"nearby" renewable energy sources (with -nationalspecification of "nearby") are included in the renewable energy contribution or not.

- Policy factors (e.g. Primary energy factors). The choice of PE factors for electricity versus e.g. gas and oil will have a direct effect on the competitiveness of technologies that use the one or the other energy carrier.

EN ISO 52003-1 (On EP indicators, requirements and ratings, [10]):

- Standardized tables for reporting in a structured and transparent manner the choices that are to be made with respect to overall EPB requirements. For example: choice on the numerical EP indicator and the EP rating method (classes).

The tables are non-restrictive, thus allowing for full regulatory flexibility. The aim is to offer choices together with the rational (motivation, pro or con) behind each choice:

- To offer possibility for harmonization.

- To bring in more transparency (comparison, exchange of best practices).

The EPB procedures are very refined (many standards, dealing with all kind of details), so it would be unproductive if the energy performance requirements are formulated in a (too) simple crude way, which would be not cost optimal or cost effective for many buildings ...

EN ISO 52010-1 (On conversion of climatic data for energy calculations, [11]):

- The weather station and climatic data set.

- Method to estimate direct solar (beam) irradiance if not available from weather station (needed for conversion to tilted and vertical planes and for calculating solar shading by external obstacles).

- Solar reflectivity of the ground (fixed value or e.g. function of snow coverage).

- (Default) solar shading from surroundings (horizon) included or not.

EN ISO 52016-1 (On the energy need for heating and cooling and indoor temperatures, [12]):

- Main choice: hourly and/or monthly method (choice may differ per category of buildings).

- Second main choice: specific rules for thermal zoning (in 10 steps); each step can be modified or replaced.

- Other typical national choices:

- Options of thermally unconditioned zone types and default values for simplifications.

- Choice between calculations with thermally coupled or uncoupled thermal zones.

- Details such as: convective fractions, solar absorption coefficient of external opaque surfaces, view factor to the sky, etc.

- Rules for operation of solar shading devices.

- Choices between options and methods for calculation of shading by external objects.

- Additionally for the hourly method only:
- Choice between a few specifically allowed alternative choices in modelling (without compromising reproducibility and transparency).

- Additionally for the monthly method only:

- The values of various correlation factors (gain utilization factors, intermittency, ...

- The parameters for effect of movable shading devices, simplified (fixed) shading calculation, ...

EN ISO 52018-1 (On partial EP indicators to building fabric and building thermal balance, [13]):

- Similar as for EN ISO 52003-1. For instance choices to set requirements on partial energy performance features (with optional choices on further details). Such as yes or no minimum requirements on one or more of the following aspects:

- Summer thermal comfort.

- Winter thermal comfort.

- Energy needs for heating and/or cooling (with further specification of assumed ventilation, etc.).

- Thermal insulation of envelope and/or individual elements.

- Thermal bridges.

- Windows energy performance.

- Air tightness.

- Solar control.

\section{Hourly calculation procedures}

\subsection{Overarching EPB standard}

The overarching EPB standard (EN ISO 52000-1, [9]) lists different options for the time interval for the calculation of the energy performance: hourly, monthly, seasonal, yearly and bin. In most countries a choice is made between monthly and hourly calculation procedures.

Overall consistency is needed in the choice of time interval for the successive modules/elements in the calculation (see fig. 1). This, however, does not mean that the choice for an hourly time interval implies that every calculation element (such as the $U$-value of constructions) is assumed to be varying on an hourly basis.

\subsection{Balanced accuracy}

For use in the context of building regulations it is essential that the procedures to calculate the energy performance of a building are not only accurate, but also robust (applicable to a wide range of cases). It is also essential that they are reproducible (unambiguous) as well as transparent and verifiable (e.g. for municipalities, to check compliance with national or regional minimum energy performance requirements) and applicable/affordable (e.g. for inspectors, assessing the energy performance assessment of an existing building). 
The accuracy of the model should always be in proportion with the limits and uncertainty in input data and with the required robustness and reproducibility of the method, but at the same time the calculation should provide a sufficient and realistic appreciation of the wide variety of available energy saving technologies: a balanced accuracy.

This concept was introduced more than a decade ago by B. Poel ([16]). It implies that the most accurate, complete and state of the art method is not necessarily the most appropriate method for a specific application. This is in particular true for calculations in the context of building regulations.

\subsection{Low energy use: strong dynamic effects}

On the other hand, many technologies, in particular for low energy buildings aiming to meet today's energy performance requirements, are strongly and dynamically interacting with the hourly and daily variations in weather and operation (solar blinds, thermostats, needs, occupation, accumulation, mechanical ventilation, night time -free cooling- ventilation, weekend operation, heat pump, solar panels, etc.). This has a strong effect on the calculated energy performance.

In the past, the dynamic effects had less prominent effects (fig. 2, from [12]). But in low energy buildings these effects can become very large (Fig. 3). This strongly influences the pro's and con's of the monthly versus hourly calculation method.

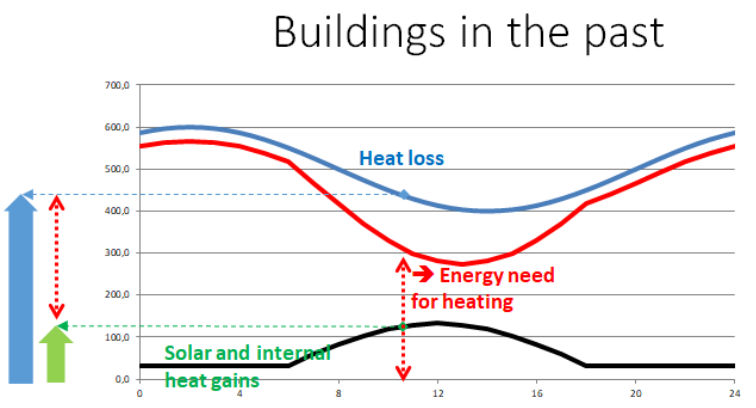

Fig. 2. Illustration of the thermal balance in case of buildings in the past: the difference between the heat losses and the heat gains ( $\sim$ the energy need for heating) is large and much less fluctuating as in low energy buildings (compare Fig. 3)

\section{Low energy buildings}

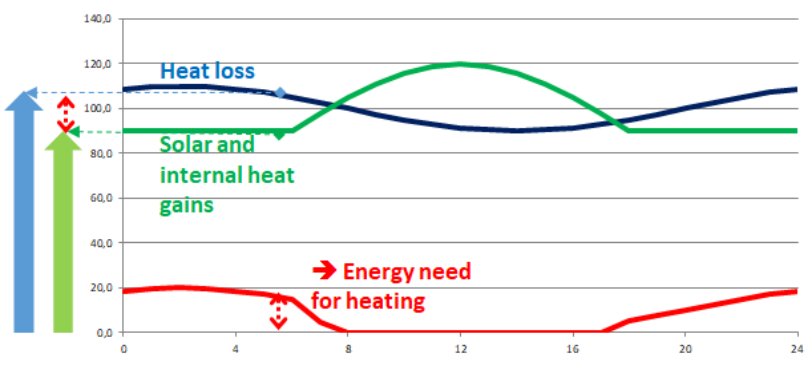

Fig. 3. Illustration of the thermal balance in case of low energy buildings: the difference between the heat losses and the heat gains ( $\sim$ the energy need for heating) is small and more fluctuating

\subsection{Monthly versus hourly method in EN ISO 52016-1}

\subsubsection{EN ISO 52016-1:2017: successor of EN ISO 13790:2008}

The choice between hourly or monthly calculation procedures is most prominently visible in the calculation of the energy needs for heating and cooling and indoor temperatures: EN ISO 52016-1 from 2017 ([12]). This standard superseded the well-known EN ISO 13790 from 2008. Like its predecessor, EN ISO 52016-1 contains, side by side, both a monthly and an hourly calculation method.

\subsubsection{Monthly method in EN ISO 52016-1}

The monthly calculation method in EN ISO 52016-1 has not been fundamentally changed compared to EN ISO 13790:2008. It contains correction or adjustment factors to account, in a kind of statistical way, for the dynamic effects that are mentioned in 5.3 above. These factors are usually pre-calculated, based on a large series of building simulations with e.g. variations of daily weather, conditions of use and building design.

However, for low energy buildings and buildings with dynamically (inter-)acting technologies, the monthly method is no longer the simple transparent method that it used to be. Due to the necessity to introduce an increasing number of correction or adjustment factors, the original transparency and robustness of the monthly method has been lost. The more of the above mentioned dynamic technologies and processes are included in the monthly calculation method, the less transparent the monthly calculation method becomes, and the more an hourly method becomes the transparent alternative.

\subsubsection{Hourly method in EN ISO 52016-1}

An hourly calculation method can directly calculate the effect of dynamic interactions and, consequently, it does not need the series of correction factors that the monthly method requires. But the challenge for an hourly method is to avoid the need for too many input data from the user. More input data would introduce more uncertainties that could easily lead to a loss of overall accuracy, or lead to significantly higher assessment costs.

The hourly calculation method in EN ISO 52016-1 has been improved drastically compared to its predecessor (EN ISO 13790:2008) in two ways:

(1) EN ISO 13790:2008 contained a very simple, aggregated (few lumped parameter nodes) model in which all building elements surrounding a thermal zone (except windows) were aggregated to a single overall thermal transmittance, including such different elements as roofs, walls and ground floor... This is actually a loss of available information $(U$ value, size, orientation and mass of each building 
element) and -consequently- to problems in e.g. the estimation of the effective thermal mass and effective solar energy gains.

In contrast to this, EN ISO 52016-1 contains a full dynamic method, in which the $U$-value, size, orientation and mass of each building element are used directly, without aggregation.

(2) At the same time, the details of this hourly method in EN ISO 52016-1 have been tailored to the goal: one of the main accomplishments of this new hourly method is that the method does not require extra input from the user compared to the monthly calculation method.

In short: the hourly and the monthly method in EN ISO 52016-1 are closely linked: they have been developed side by side and they use, all together, the same input data and assumptions.

\subsubsection{Hourly method in EN ISO 52016-1 to derive monthly correlation factors}

Consequently, the hourly method is also very well suited for the derivation or validation of the correction and correlation factors of the monthly method. For instance, by carrying out a large number of hourly calculation runs for a specific range of building categories, with a variety of building types and designs. This is illustrated by the flow chart in figure 4.

Normally, another dynamic simulation tool is needed for such purpose; with as a consequence that differences in assumed conditions and differences in model approaches lead to a lot of noise in the derivation/validation of the correction or correlation factors. This is eliminated by comparing the side by side developed hourly and monthly method from EN ISO 52016-1.

\subsubsection{Link with EN ISO 52010-1}

One of the additional causes for the kind of "noise" mentioned above is the amount of solar irradiation at vertical and tilted planes, calculated on the basis of the measured solar irradiation at horizontal plane. This is an important input for the calculation of energy needs and indoor temperatures, especially in case of low energy buildings.

The main internationally available building simulation tools use quite similar but yet different algorithms for this conversion. This is probably one of the reasons why in the so called BESTEST cases ([18]) discrepancies between building simulation tools occur on the calculation of energy needs for heating and cooling and indoor temperatures. With the introduction of EN ISO 52010-1 [11], this algorithm has now been harmonized which eliminates this additional noise. This is explained in more detail in 7.2.1 of the Technical Report accompanying EN ISO 52016-1 [19].

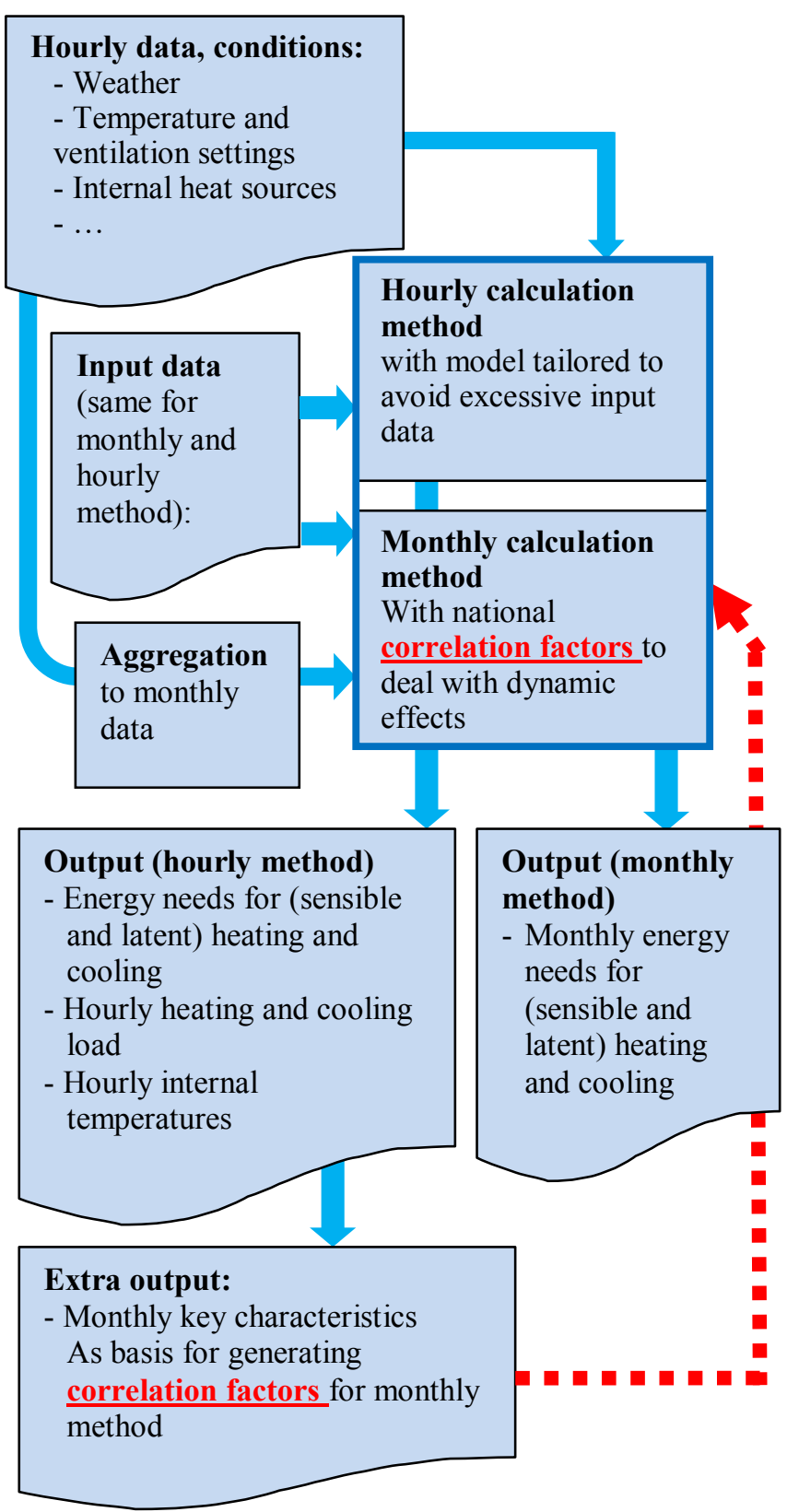

Fig. 4. EN ISO 52016-1: links between the hourly and the monthly method provided in this standard

\subsection{Smart readiness}

One could easily defend that the assessment of the smart readiness of a building, one of the new concepts in the revised EPBD [1], requires hourly (or sub-hourly) calculation intervals. It is quite evident that in different buildings the same "smart" service or technical feature can result in different outcomes. According to the revised EPBD ([1]) the 'Smart Readiness Indicator' (SRI) should focus on the building's adaptation to user and grid needs. Only assessment of the performance of the building by calculation or measurement can provide a realistic quantification of the 'smart readiness'.

Evidently, a monthly calculation method lacks the possibility to reveal the potential to adapt to the user and grid needs. In fact, the use of monthly average data (e.g. monthly mean electricity surplus or shortage), intrinsically leads to over-optimistic results. The set of 
EPB standards offer the possibility for hourly calculations to provide meaningful results.

\section{Service Contract with European Commission}

The European Commission awarded a service contract (Sept.2018 - Sept. 2021) to a team led by ISSO ([17]), to support the uptake of the EPB standards. Examples of activities under this contract:

- Support to EU Member States and National Standardization Bodies that wish to complete the National Annexes of the EPB standards.

- Set up a public frequently asked questions database on filling in National Annexes, on practical application of the standards, etc.

- Preparation of practical case studies to support the use of standards and dissemination and development of calculation tools for individual standards.

- Set up of a large network of practitioners and support of the uptake of the EPB standards by organising regular hands-on workshops, training sessions, etc.

The EPB Center, founded by ISSO and REHVA, will be the communication platform to offer these services. More information will gradually become available at the website (www.epb.center). The web site also offers the possibility to provide feedback, suggestions and questions on the set of EPB standards and their implementation.

\section{Conclusions}

With the publication of the set of EPB standards (2017) a powerful set of internationally harmonized assessment procedures on the energy performance of buildings has become available. These harmonized procedures offer flexibility to take into account the national or regional situation via so called national annexes or datasheets.

The EPB Center provides information and technical support for and collects feedback on the uptake of the set of EPB standards at national and regional levels.

This set of methods is a key instrument to set and evaluate the national and international policy targets and will play an important role in driving innovation in the construction sector.

\section{References}

1. EPBD:2018, Directive (EU) 2018/844 of 30 May 2018 amending Directive 2010/31/EU on the energy performance of buildings and Directive 2012/27/EU on energy efficiency. (2018)

2. Mandate $\mathrm{M} / 480$, Mandate to CEN, CENELEC and ETSI for the elaboration and adoption of standards for a methodology calculating the integrated energy performance of buildings and promoting the energy efficiency of buildings, in accordance with the terms set in the recast EPBD (2010/31/EU), December 14, 2010 (2010)
3. CEN/TS 16628, Energy Performance of Buildings Basic Principles for the set of EPB standards (subject to revision) (2014)

4. CEN/TS 16629, Energy Performance of Buildings Detailed Technical Rules for the set of EPB standards (subject to revision) (2014)

5 The EPB Center information and communication platform, www.epb.center

6 J. Hogeling (ed.), Special issue "EPB standards", 53, 3 (2016)

7 J. Hogeling (ed.), Special issue "EPB standards published for formal vote", REHVA Journal, 53, 6 (2016)

8 J. Hogeling, The implementation of the new EPBstandards will boost product and HVAC system innovation and create new market opportunities for the HVAC industry, editorial REHVA Journal, 54, 1 (2017).

9 EN ISO 52000-1, Energy performance of buildings - Overarching EPB assessment - Part 1: General framework and procedures (2017)

10 EN ISO 52003-1, EPB - Indicators, requirements, ratings and certificates - Part 1: General aspects and application to the overall energy performance (2017)

11 EN ISO 52010-1, EPB - External climatic conditions - Part 1: Conversion of climatic data for energy calculations (2017)

12 EN ISO 52016-1, EPB - Energy needs for heating and cooling, internal temperatures and sensible and latent heat loads - Part 1: Calculation procedures (2017)

13 EN ISO 52018-1, EPB - Indicators for partial EPB requirements related to thermal energy balance and fabric features - Part 1: Overview of options (2017)

14 D. van Dijk, Spotlight on the EN ISO 52000 family of EPB standards, REHVA Journal, 54, 6 (2017)

15 D. van Dijk, EPB standards: Why choose hourly calculation procedures?, REHVA Journal, 55, 1 (2018)

16 B. Poel, Contribution to Applying the EPBD to improve the Energy Performance Requirements to Existing Buildings - ENPER-EXIST, Final Report of WP1 of ENPER-EXIST, (2007)

17 Service Contract $N^{0}$ ENER/C3/2017-437/ SI2.785185 with the European Commission, DG Energy (Sept. 2018 - Sept. 2021), Support the Dissemination and Roll-out of the Set of Energy Performance of Building Standards developed under EC Mandate M/480. Coordination and contact: Jaap Hogeling, ISSO (NL) (2018)

18 ANSI/ASHRAE standard 140, Standard Method of Test for the Evaluation of Building Energy Analysis Computer Programs (2014)

19 CEN ISO/TR 52016 2:2017, Energy performance of buildings - Energy needs for heating and cooling, internal temperatures and sensible and latent heat loads - Part 2: Explanation and justification of ISO 520161 and ISO 520171 